\title{
Interpretation of the Behaviour of Compacted Soils Using Cam-Clay Extended to Unsaturated Conditions
}

\author{
Francesca Casini ${ }^{1}$, Roberto Vassallo ${ }^{2}$, Claudio Mancuso ${ }^{3}$, and Augusto \\ Desideri $^{1}$ \\ 1 Dipartimento di Ingegneria Strutturale e Geotecnica, Università degli Studi di \\ Roma "La Sapienza", Italy \\ francesca.casini@uniroma1.it, augusto.desideri@uniroma1.it \\ 2 Dipartimento di Strutture, Geotecnica, Geologia Applicata all'Ingegneria, \\ Università degli Studi della Basilicata, Italy roberto .vassallo@unibas .it \\ 3 Dipartimento di Ingegneria Geotecnica, Università degli Studi di Napoli Federico \\ II, Italy claudio.mancuso@unina.it
}

Summary. This paper is focused on the interpretation of experimental results obtained at the Department of Geotechnical Engineering of the University of Naples Federico II (Italy) to investigate the effects of partial saturation on the volumetric behaviour and the initial shear stiffness of a compacted silt. Tests were performed by using suction-controlled triaxial and resonant column cells. Herein, the compatibility of the results with a Single Stress Model (SSM) is discussed. The SSM allows to highlight that suction can have two effects on the mechanical behaviour of an unsaturated soil: it increases the average volumetric stress acting on the soil skeleton and it has a sort of cementing effect on the soil packing (hardening and cementation).

Key words: compacted soil, constitutive modelling, single stress variable, elastoplastic, compressibility, small strain behaviour

\section{Introduction}

The experimental results obtained at the University of Naples Federico II (Italy) for the characterization of the small and moderate strain-behaviour of a compacted silt (Vassallo et al. 2006a) are interpreted in this paper using a Modified Cam Clay Model extended to unsaturated conditions (Jommi and di Prisco 1994, Tamagnini 2004).

The tested soil is representative of the materials used for constructing embankments on the Po river (Italy). It has a liquid limit $w_{L}=50.4 \%$ and a plasticity index $I P=17.9 \%$. The soil was compacted at the optimum water content with the standard Proctor procedure (ASTM D691-91). On average, $w=23.12 \%$ and $\gamma_{d}=15.60 \mathrm{kN} / \mathrm{m}^{3}$ were obtained. 
All tests included an equalization, during which suction changed from the after compaction value to the desired value, at mean net stress $p-u_{a}$ of about $10 \mathrm{kPa}$. Then, the imposed suction was kept constant during compression. Finally, in some cases, wetting and/or drying stages at constant $\left(p-u_{a}\right)$ were carried out (Vassallo et al. 2006a).

Experimental results were analysed by Vassallo et al. (2006b) using two independent stress variables and the framework of hardening plasticity.

\section{Modelling Framework}

The constitutive laws for the mechanical behaviour are defined in terms of effective stresses. The average soil skeleton stress $p^{\prime}$ is defined as the difference between total stress and an equivalent fluid pressure, with the degree of saturation as weighing parameter:

$$
p^{\prime}=p-u_{a}+S_{r}\left(u_{a}-u_{w}\right) .
$$

The evolution of the scalar internal variable $p_{c}^{\prime}$ (overconsolidation pressure) depends not only on the rate of plastic strains but also on the variations of degree of saturation:

$$
\dot{p}_{c}^{\prime}=\dot{p}_{c \text { sat }}^{\prime}-b^{\prime} p_{c} \dot{S}_{r}
$$

where $b$ is a constant soil property. The integration of Eq. (2) yields to:

$$
p_{c}^{\prime}=p_{c \text { sat }}^{\prime} \mathrm{e}^{b\left(1-S_{r}\right)} .
$$

Thus, $b$ controls the rate of change in $p_{c}^{\prime}$ caused by variations in $S_{r}$.

Hardening has an irreversible component dependent on the development of plastic volumetric strains, related to the evolution of $p_{c \text { sat }}^{\prime}$, and a reversible component related to changes in $S_{r}$.

The model predicts that a drying process induces some bonding and positive hardening while a wetting process induces some debonding (negative hardening), as shown in Fig. 1.

Let us consider an isotropic test in which a soil sample is subjected to an increment of $p^{\prime}$ at the virgin state. Similarly to saturated soils, the specific volume $v=1+e$ will be given by:

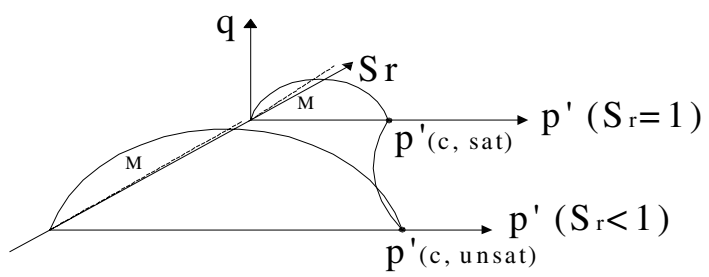

Fig. 1. Evolution of the hardening surface in the space $p^{\prime}: S_{r}: q$ 


$$
v=N\left(S_{r}\right)-\lambda \ln p^{\prime}
$$

where $p^{\prime}$ is the stress defined by Eq. (1) and $N\left(S_{r}\right)$ is the specific volume at $p^{\prime}=1 \mathrm{kPa}$.

The relationship which defines how $N$ changes with the degree of saturation can be obtained from previous equations and from:

$$
\begin{aligned}
& v_{s}=N-\lambda \ln p_{c \text { sat }}^{\prime}, \\
& v_{s}=v-\kappa \ln \frac{p_{c \text { sat }}^{\prime}}{p_{c}^{\prime}}
\end{aligned}
$$

where $v_{s}$ is the specific volume of the saturated soil, $N$ (equal to 2.01 for the considered soil) is the specific volume at $p^{\prime}=1 \mathrm{kPa}$ in saturated conditions. By substitution, one obtains:

$$
N\left(S_{r}\right)=N+(\lambda-\kappa) b\left(1-S_{r}\right)
$$

If there is a variation of degree of saturation, this corresponds to a translation of the Normal Consolidation Line, parallel to the saturated NCL (Fig. 2). The amount of translation is related to the constitutive parameter $b$. By interpreting all the available compression stages (25, in total), an average value of 7 was obtained.

The model requires an hydraulic constitutive relationship describing the water storage mechanism, such as $S_{r}=W R C(n s)$ (Houlsby 1997, Tamagnini 2004), where $n s$ is the product of soil porosity and suction. In this study, a modified form of the equation proposed by Van Genuchten (1980) is used:

$$
S_{r}=\left[\frac{1}{1+(\alpha n s)^{r}}\right]^{t}
$$

whit $r=1, t=0.8$, and:
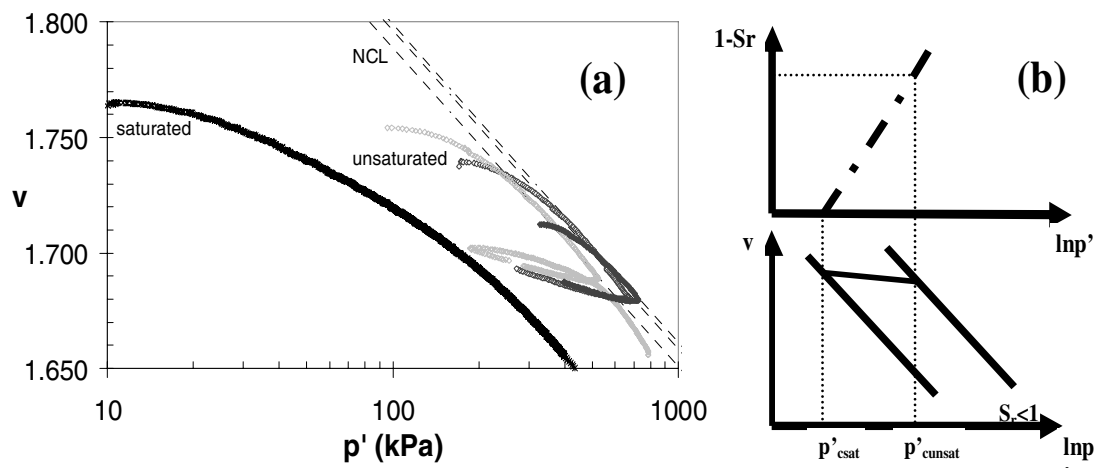

Fig. 2. (a) Position of the NCL at various $S_{r}$, (b) prediction for isotropic state 


$$
\alpha=A \exp \left(B n_{0}\right)
$$

with $A=6 \times 10^{-09} \mathrm{kPa}^{-1}$ and $B=40$. In Eq. (8), $n_{0}$ is the porosity at the end of equalization. Such dependency was chosen to isolate the effect of the imperfect homogeneity of after compaction porosities of the tested specimens, which affect after-equalization values (Vassallo et al. 2006a).

Thus, a single relationship was assumed for $S_{r}$ variations, without taking into account possible differences between wetting and drying paths. Certainly, this will have to be considered in the continuation of the study.

\section{Interpretation of Results at Moderate Strains}

In Figure 3 the experimental results of three compression stages (loading and unloading) are compared with model predictions. It is worth noting that, also for most of other compressions, the model predicts an overconsolidated state at the end of equalization, according to experimental data. A good qualitative and quantitative correspondence is found in general.

Figure 4 is relative to two tests including suction cycles.

Test mp05RC included a compression at $s=200 \mathrm{kPa}$, up to $p-u_{a}=200 \mathrm{kPa}$ and suction cycles between 100 and $400 \mathrm{kPa}$. The model (Fig. 4) overestimates the plastic strains due to compression and underestimates the strains along the first drying at $s=400 \mathrm{kPa}$. This is due to the chosen slope of WRC which, in this case, leads to overestimate the reduction in $S_{r}$ due to the drying process. Because of this, the model predicts an excessive reversible component of hardening and underestimates plastic strains. The subsequent wetting and drying stages only induce reversible strains, according to experimental data. Better predictions could be probably achieved by taking into account the hydraulic hysteresis of the WRC.

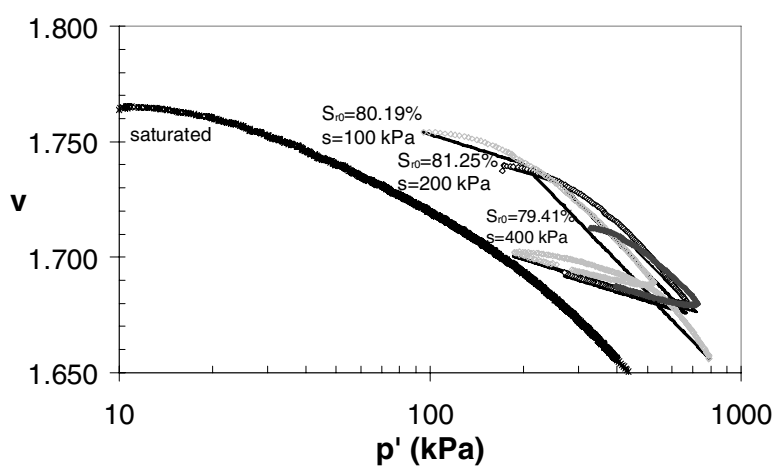

Fig. 3. Comparison between experimental results and model predictions for compression tests carried out at three different suction levels 


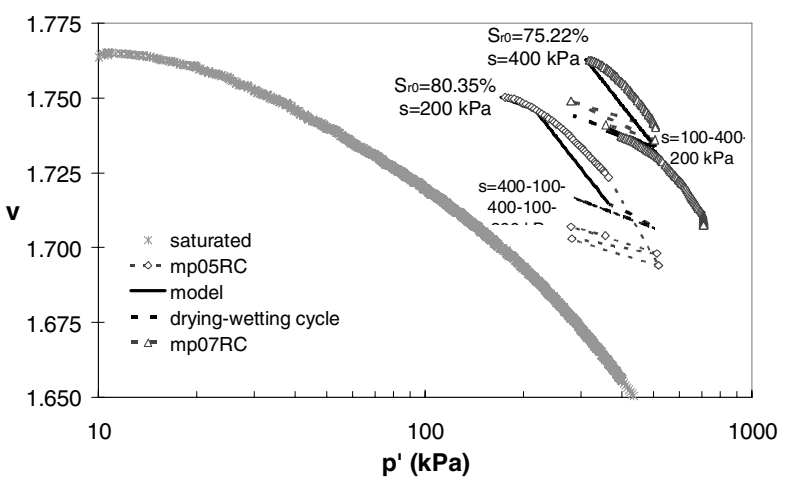

Fig. 4. Comparison between experimental results and model predictions for tests including compression and drying-wetting stages

Test mp07RC included a compression at $s=400 \mathrm{kPa}$, up to $p-u_{a}=$ $200 \mathrm{kPa}$ and suction cycles between 100 and $400 \mathrm{kPa}$. The model predicts normal consolidation at the beginning of the test, while experimental results in the plane $v-p^{\prime}$ show a light overconsolidation. Predictions relative to the following wetting, drying and compression stages are in good agreement with experimental data.

\section{Interpretation of Results at Small Strains}

The proposed model can also be used as a framework to interpret the variations of initial shear stiffness $G_{0}$ with mean net and suction observed by Vassallo et al. (2006a).

Before proposing such interpretation, it is worth recalling some features of saturated soil behaviour. Initial stiffness depends on the average effective stress $p^{\prime}$. Rampello et al. (1994), examining data relative to reconstituted and undisturbed materials, showed that an undisturbed material, thanks to its "structure," has higher values of $G_{0}$ than the same material in a reconstituted state (Fig. 5). The effect of this structure is lost as an effect of increasing $p^{\prime}$. Furthermore, reconstituted normally consolidated and overconsolidated materials show $G_{0}: p^{\prime}$ curves qualitatively similar to the curves $v: p^{\prime}$ in the compressibility plane. A linear relationship can be assumed between $\log G_{0}$ and $\log p^{\prime}$ for both normally and over-consolidated states, with a lower slope for overconsolidation.

Using same representations as Rampello et al. (1994), $G_{0}$ values measured under controlled suction conditions by resonant column tests have been plotted in Fig. 5a as a function of the stress $p^{\prime}$ defined by Eq. (1). Moving from complete saturation to partial saturation induces a translation upwards of experimental curves $G_{0}: p^{\prime}$, similar to that highlighted by Rampello et al. (1994) 

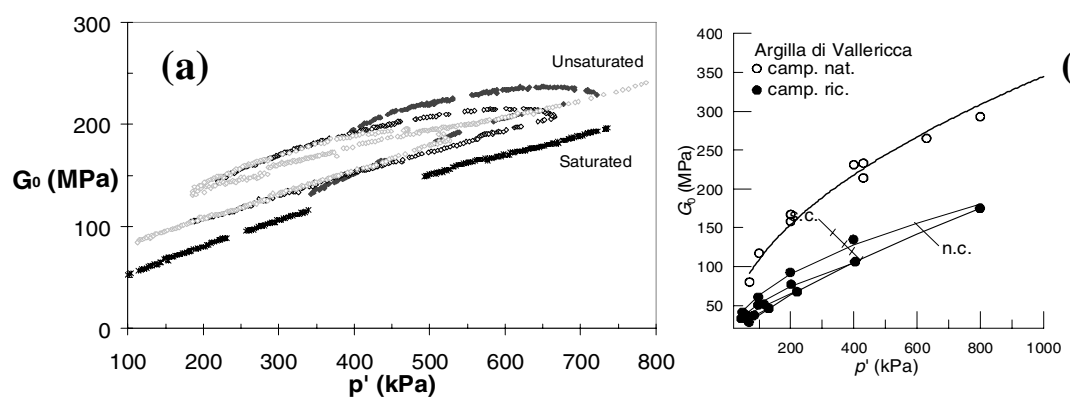

Fig. 5. Comparison: (a) saturated-unsaturated compacted soil, (b) naturalreconstituted saturated soil

comparing the behaviour of natural and reconstituted clays. Partial saturation seems to provide the solid skeleton with a structure (or cementation) similar to ageing and other natural time effects. In addition, experimental points relative to measurements of $G_{0}$ at beginning of the tests show trends similar to that of an overconsolidated saturated soil.

This is in agreement with the observations made above for the end of equalizations: the soil results overconsolidated also in the compression plane $v: p^{\prime}$. All the above suggests that a reversible phenomenon of "bonding" is associated to a drying path.

A first attempt to interpret this phenomenon was made by considering the hardening predicted by the SSM.

The relationships proposed by Rampello et al. (1994) for saturated soils was extended by including the effects of $S_{r}$ on void ratio and on $p^{\prime}$ :

$$
G_{0}=S^{*} p_{r}^{\left(1-n^{*}+c\right)} f(e) p^{\left(n^{*}-c\right)}
$$

where

$$
f(e)=C / \exp (D e)
$$

and: $c=m / \Lambda, \Lambda=(\lambda-\kappa) / \lambda, C=\exp \left[c\left(N\left(S_{r}\right)-1\right) / \lambda\right], D=c / \lambda ; p_{r}$ is a reference pressure.

Parameters $S^{*}, n^{*}$ and $m$ (linked to the position and the slope of $\log G_{0}$ : $\log p^{\prime}$ relationships) were obtained by fitting the saturated soil data (Fig. 6). Resulting values are $n^{*}=0.61, m=0.2, S^{*}=3000$.

It was found that Eqs. (9) and (10) significantly underestimate experimental stiffness of the unsaturated soil. Thus, the extension of saturated relationships to the unsaturated case requires further assumptions. A better agreement can be obtained by supposing that also $n^{*}$ and $S^{*}$ depend on $S_{r}$. The obtained results are shown in Fig. 6 for the same tests of Fig. 3. It was found that $n^{*}$ decreases and $S^{*}$ increases as $S_{r}$ decreases. The values used in the figure are $n^{*}=0.60$ and $S^{*} \cong 4300$ for $S_{r, 0} \cong 85 \%$ (Fig. $6 \mathbf{a}$ ), $n^{*}=0.59$ and $S^{*} \cong 4500$ for $S_{r, 0} \cong 82 \%, n^{*}=0.59$ and $S^{*} \cong 4800$ for $S_{r, 0} \cong 79 \%$ (Fig. 6b). 

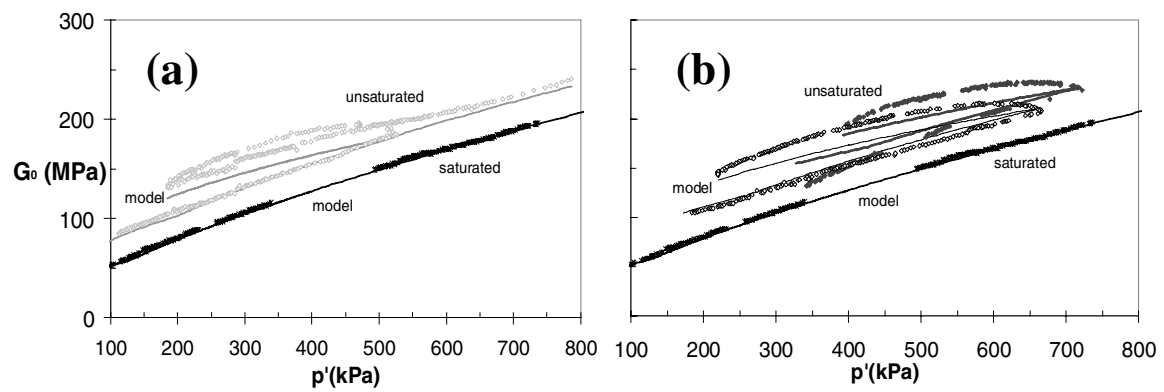

Fig. 6. Comparison between experimental results and model predictions of initial shear stiffness $G_{0}$. (a) compression test carried out at $s=100 \mathrm{kPa},(\mathbf{b})$ compression test carried out at $s=200$ and $400 \mathrm{kPa}$

\section{Conclusions}

In this paper it was verified if the comprehensive experimental study on the Po silt by Vassallo et al. (2006a) can be interpreted within the framework of a Single Stress Model (SSM).

Such model can interpret the progressive shift in the plane $v: p^{\prime}$ of the normal consolidation line as the degree of saturation decreases and, more in general, the influence that $S_{r}$ has on compressibility.

The effects of partial saturation on the initial shear stiffness $G_{0}$ are similar to those ascribable to the structure of a natural soil, compared to the same soil reconstituted. They cannot be justified by simply taking into account the effect that the SSM predicts on the void ratio without significantly underestimating the unsaturated soil stiffness. On the contrary, it is necessary to consider explicitly the effect that $S_{r}$ has on some parameters describing the relationship $G_{0}: p^{\prime}$.

This is a preliminary study, and some simplifications, such as neglecting the hydraulic hysteresis, will have to be removed in the continuation. However, the results thus far obtained seem encouraging.

\section{References}

Houlsby GT (1997) The work input to an unsaturated granular material, Geotechnique 47(1):193-196

Jommi C, di Prisco C (1994) A simple theoretical approach for modelling the mechanical behaviour of unsaturated soils. In: Italian Conference, Il ruolo dei fluidi nei problemi di ingegneria geotecnica, Mondovi, pp 167-188

Rampello S, Silvestri F, Viggiani G (1994) The dependence of small strain stiffness on stress state and history for fined grained soils: the example of Vallericca clay. In: Proc 1st Int Symp on Pre-Failure Defomation of Geomaterials, Sapporo, pp 273-278 
Romero E, Vaunat J (2000) Retention curves of deformable clay. Experimental evidence and theoretical approaches in unsaturated soils, Trento, pp 91-106

Tamagnini R (2004) An extended Cam-clay model for unsaturated soils with hydraulic hysteresis, Géotechnique 54:223-228

Van Genuchten MTh (1980) A closed form equation for predicting the hydraulic conductivity of unsaturated soils, Soil Sci Soc Amer J 44(5):892-898

Vassallo R, Mancuso C, Vinale F (2006a) Effects of net stress and suction history on the small strain stiffness of a compacted clayey silt. Can Geotech J (accepted)

Vassallo R, Mancuso C, Vinale F (2006b) Modelling the influence of stress-strain history on the initial shear stiffness of an unsaturated compacted silt, Can Geotech J (accepted) 